\title{
Miscibility gap of octyl glucoside-phosphatidylcholine micellar solutions.

\author{
Partition of the nicotinic acetylcholine receptor \\ into the surfactant-rich phase
}

\author{
Theo Schürholz, Anke Gieselmann and Eberhard Neumann \\ Department of Physical and Biophysical Chemistry, Faculty of Chemistry. Unicersitv of Bielefeld. Bielefeld (F.R.G.)
}

(Received 8 May 1989)

Key words: Vesicle; Micelle; Octyl glucoside; Phosphatidylcholine: Protein reconstitution: Protein purification; Acetylcholine receptor; Nicotinic acetylcholine receptor

Phospholipid phosphatidylcholines ${ }^{\prime} \mathcal{C}$ ) and the nonionic detergent octyl glucoside (OG) were found to form a miscibility gap in aqueous solution at a molar ratio $|O G|_{T} /|P C|_{T}=2,6-2.4$. This ratio is just below the sritical ratio for the formation of vesicles. At $[P C]>1.3 \mathrm{~g} / \mathrm{l}$ the transition from micelles to vesicles is not direct but via a surfactant-rich phase (SRP). The various stability regions are summarized in a phase diagram. Though the partition coefficient $\Gamma=\left[D_{b}\right] /\left[D_{w}\right]$ increases markedly with temperature, the critical ratio for the phase separation $R^{c}$ is biased to higher detergent concentrations. This behaviour can be explained by increased motion of the hydrocarbon moiety at higher temperatures. The different phases are investigated by light microscopy, nuclear magnetic resonance and thin-layer chromatography. The miscibility gap of PC/OG is compared to the lamellar phase of Triton X-114; the miscibility gap has implications for membrane protein reconstitution and can be used for membrane protein purification. The Torpedo califomica nicotinic acetylcholine receptor (n-AcChR) was found to partition into the SRP.

\section{Introduction}

The use of detergent to solubilize biological membranes was an essential and necessary step in the investigation of membrane proteins [1,2]. If, the detergent is afterwards removed by dialysis or gel chromatography, the dissolved proteins can be investigated in an artificial, reconstituted membrane system. In this context the function of membrane channel proteins may be studied by vesicle flux [3] or by electric current measurements across planar lipid bilayers $[4,5]$.

Although detergents are commonly applied in membrane biochemistry, the mechanism of membranemicelle transformation has only been studied recently (Refs. 6-11 and references therein). The main result is

\footnotetext{
Abbreviations: $\mathbf{n - A c C h R}$, nicotinic acetylcholine receptor; $\mathbf{O G}, n-o c-$ tyl $\beta$-D-glucopyranoside; SRP, surfactant-rich phase; LMV, large multilamellar vesicles.
}

Correspondence: T. Schürholz, Department of Physical and Biophysical Chemistry, Faculty of Chemistry, University of Bielefeld, P.O. Box 8640, D.4800 Bielefeld 1, F.R.G. that the ratio of detergent to lipid in the membrancs as well as in the micelles is decisive for the structural transition.

Bilayer-vesicles and micelles are only two of the many possible lyotropic structures (or mesophases) which are known to occur in surfactant colloidal suspensions or emulsions. In addition, some of the structures, e.g. vesicles, are metastable mesophases; these phases can depend on the way of mixing and on the direction of temperature change. The structural reorganizations are often so slow that the equilibrium state is not attained and is thus overlooked. Since microemulsions and mesomorphic structures have been discovered as matrices for enzyme-coupled reactions, understanding and controlling of such colloidal systems will be also important for the development of this new branch of biotechnology [12].

The phospholipid phosphatidylcholine (PC) is often used to prepare model membranes. The main advantages cf $\mathrm{PC}$ are its natural abundance and its good chemical stability. The nonionic detergent $n$-octyl $\beta$-D-glucofyranoside $(O G)$ is frequently applied in biochemistry to solubilize and reconstitute membrane proteins. It has 
a high critical micelle concentration (CMC) which is advantageous for its removal afterwards. $O G$ is mild with respect to the denaturation of proteins.

Nonionic detergents like poly(glycol ethers) frequently show a miscibility gap at elevated temperatures. The critical temperature of this two-phase region, called cloud point is rather moderate for Triton X-114 (about $28^{\circ} \mathrm{C}$ ). The surfactant-rich phase nas a lamellar structure [13] and was proposed as a matrix for the study of membrane proteins. Due to their different solubilities in the two phases, hydrophobic membrane proteins generally partition into the surfactant-rich phase and therefore can be separated from water-soluble proteins [14]. Recently, a miscibility gap has been reported for mixtures of $O G$ and $P C[9,10]$.

Here we have investigated the OG.PC miscibility gap with respect to vesicle formation and discuss its implications on the reconstitution of membrane proteins. In particular, the up to now unsatisfying results of the reconstitution of Torpedo californica nicotinic acetylcholine receptor [15] have necessitated the careful study of protein/lipid vesicle formation from protein/ detergent micelles. Here, we show that the formation of a macroscopic phase impairs the reconstitution process, but can also be used to monitor the progress of vesicle formation.

\section{Materials and Methods}

1,2-Dioleoyl-sn-glycero-3-phosphocholine (DOPC), 1-palmitoyl-2-oleoyl-sn-glycero-3-phosphocholine (POPC), dimyristoyl-sn-glycero-3-phosphocholine (DMPC), 1,2-dipalmitoyl-sn-glycero-3-phosphoserine (DPPS) and PC (95\%) were purchased from Avanti. PC $(95 \%)$ is a soybean phosphatide extract with $95 \%$ phosphatidylcholine. $n$-Octyl $\beta$-D-glucopyranoside (OG) was from Sigma. Acetylcholine receptor protein (AcChR) from Torpedo californica electric organ tissue was purified by affinity chromatography according to Refs. 16 and 17 using (3-[(3-cholamidopropyl)dimethylammonio]-1-propanesulfonate) (CHAPS) as detergent (1\%). The experiments were performed in $10 \mathrm{mM}$ piperazine$N, N^{\prime}$-bis(ethanesulfonic acid) ((Na)Pipes), pH 7.1 and $150 \mathrm{mM} \mathrm{NaCl}$, if not stated otherwise.

Sample preparation. The lipid was added to a prepared OG solution either as powder or as suspension in buffer (large multilamellar vesicles, LMV). To accelerate the dispersion of the surfactant bath sonication was applied for one to three minutes. The samples were kept over night in small glass vials at the desired temperature before phase formation was analysed (by eye). The relative volume of the phases were measured to determine the extension of the miscibility gap. After thorough mixing (bath sonication) and incubation the phase formation was analysed a second time.

Turbidity measurements. The relative turbidity of micellar or vesicular dispersions was measured in a Perkin-Elmer MPF-44A fluorimeter at a wavelength of $500 \mathrm{~nm}$. At the beginning of a dilution experiment the [PC] was between $10 \mathrm{~g} / 1$ and $25 \mathrm{~g} / 1$, the [OG] was at least a factor of 1.5 higher. $1 \mathrm{ml}$ samples were diluted with buffer solution in steps of $10 \mu \mathrm{l}$. After each addition of buffer the sample was shaken and 5 minutes were given for equilibration. At the phase separation (indicated by a decrease in turbidity) the dilution was stopped or interrupted until the phases had separated. Complete phase separation was judged by eye, i.e., from a sharp boundary.

Thin-layer chromatography (TLC). The lipid and the detergent in the phases were analysed on HPTLC plates from Merck (F.R.G.) with chloroform/methanol/water $(65: 25: 4, v / v)$ as mobile phase and stained by iodine vapor.

Determination of phosphatidylcholine. Samples were made up to a $100 \mu 1$ volume with $\mathrm{H}_{2} \mathrm{O} .300 \mu 1$ of $60 \%$ perchloric acid were added and incubated at $220^{\circ} \mathrm{C}$ for $2 \mathrm{~h}$. Then $0.9 \mathrm{ml} \mathrm{H}_{2} \mathrm{O}, 5 \mathrm{ml}$ dye reagent (see below) and $200 \mu 11.5 \%$ Tween 80 in water were added and vortexed. The absorbance $(A)$ was measured at a wavelength of $660 \mathrm{~nm}$. The calibration curve was performed with soybean PC (95\%), Avanti.

Dye reagent: 1 part $4.2 \%$ ammonium heptamolybdate in $5 \mathrm{~mol} / \mathrm{H} \mathrm{HCl}$ and 3 parts $0.2 \%$ malachite green [18].

Determination of octyl glucoside. Samples were made up to a $100 \mu \mathrm{l}$ volume with $\mathrm{H}_{2} \mathrm{O} .1 \mathrm{ml} 0.2 \%$ anthrone (Sigma A-1631) in concd. $\mathrm{H}_{2} \mathrm{SO}_{4}$ was added and vortexed. After $1 \mathrm{~h}$ at ice temperature the samples were incubated at $90^{\circ} \mathrm{C}$ for $9 \mathrm{~min}$ and then cooled down on ice again. The absorbance was measured at $630 \mathrm{~nm} \mathrm{[19].}$

Phospholipid did not interfere with the assay.

Darkfield microscopy. The micrograph (Fig. 3) was taken on an Olympus darkfield microscope (BHS, darkfield oil immersion condenser) and flashlight illumination. The magnification of the objective was $40 \times$. The size of particles was measured by aid of an eyepiece-micrometer.

To observe vesicle formation at the phase boundary, the microscope was tilted by $90^{\circ}$. With a cover glass and three $100 \mu \mathrm{m}$ spacers a pocket was assembled on a microscope slide. An aliquot of the SRP phase was filled into the pocket and detergent-free buffer carefully laysect in ic i.

\section{Results}

Phase formation as a function of surfactant composition

When a mixed micellar suspension of $O G$ and PC was gradually diluted with buffer in order to form vesicles, the optical turbidity suddenly dropped after an initial slight increase. If then the suspension was left 
TABLE I

Critical ratio $R_{T}^{c}$ for different $P C$ concentrations and $P C$ species

$\boldsymbol{R}_{\mathrm{T}}^{\mathbf{c}}=[\mathrm{OG}]_{\mathrm{T}} /[\mathrm{PC}]_{T}$ characterizes the transition from a micellar solution to the two-phase region.

\begin{tabular}{lllll}
\hline PC & {$[\mathrm{PC}](\mathrm{g} / \mathrm{l})$} & {$[\mathrm{OG}](\mathrm{g} / \mathrm{l})$} & $R_{\mathrm{T}}^{\mathrm{c}}$ & $T\left({ }^{\circ} \mathrm{C}\right)$ \\
\hline POPC & $5.6 \pm 0.5$ & $9.1 \pm 0.5$ & 1.6 & \\
POPC & $25 \pm 1$ & $27 \pm 1$ & 1.1 & 20 \\
POPC & $42 \pm 2$ & $42 \pm 2$ & 1 & \\
POPC & 25 & 26 & 1.05 & \\
DMPC & 25 & 24 & 0.95 & 5 \\
PC95 & 22 & 27 & 1.2 & \\
\hline
\end{tabular}

unstirred, it separated into two clear phases within minutes to hours. The separation depends on composition and is faster at higher temperatures. Upon further dilution with buffer the portion (volume) of the surfactant-rich phase, SRP (the lower phase containing both lipid and detergent), decreased in favor of the formation of vesicles. Vesicle formation was indicated by a white, light scattering band at the phase boundary. The vesicle precipitate increased in size with further dilution intil the SRP had vanished. This phase behavior is illustrated in Fig. 1, where the phase areas are plotted as a function of composition and of temperature.

Whereas dilution was used to simulate the different stages of vesicle formation during a dialysis procedure the phase diagram (Fig. 1) was constructed from data where increasing amounts of $\mathrm{PC}$ were added to a micellar suspension of $O G$. The total concentration is $[\mathrm{OG}]_{\mathrm{T}}$ $=27 \mathrm{~g} / \mathrm{l}$ and was chosen because in this range the two phases are about equal in volume.

Addition of $\mathrm{PC}$ below the critical ratio $R_{\mathrm{T}}^{\mathrm{c}}=$ $[O G]_{T} /[P C]_{T}=1 . j 8\left(\right.$ at $20^{\circ} \mathrm{C}$ ) ${ }^{*}$ caused the system to divide into two macroscopic phases. The value of $R_{\mathrm{T}}^{\mathrm{c}}$ varied with the type of PC used (Table 1 ). By addition of detergent the process can be reversed. Normally, lipids were added as a suspension in buffer (large multilamellar vesicles, LMV) and solubilization was accelerated by bath sonication. Addition of PC and $O G$ in a different way, e.g. as powder, had no influence on the phase diagram. The niscibility gap with two clear phases was only existent in a sinall range of $O G / P C$ ratios: $1.08 \geq R_{\mathrm{T}} \geq 1$. At $R_{\mathrm{T}}<1$, vesicles formed at the boundary between the two phases. Simultaneously, the volume of the SRP diminished, which is indicated by the decreasing thickness of the black stripes in Fig. 1.

\footnotetext{
* Ratios of total PC and OG are indicated by the index T, e.g. $R_{\mathrm{T}}$ The numbers are given in $\omega / \omega$, if not indicated differently. $R_{T}$ (and one concentration) is used to characterize the system as a whole, i.e., the boundaries of the miscibility gap; the weight unit was taken with respect to the partial use of natural lipids. Ratios in the phases or aggregates $R$ are expressed in $\mathrm{mol} / \mathrm{mol}$. The characters in brackets indicate the type of aggregate, e.g., $R(\mathrm{SRP})$.
}

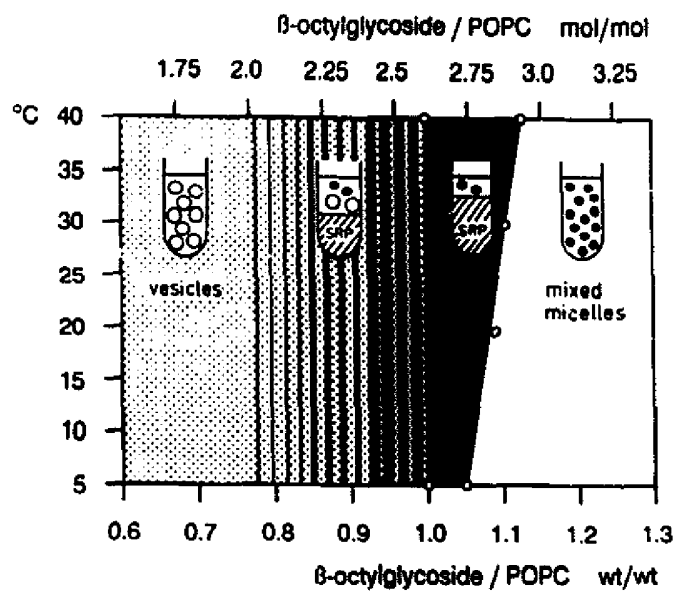

Fig. 1. Phase diagram of plosphatidylcholine (PC) and $\beta$-octyl glucosic: (OG) at the micelle/vesicle transition. Four areas can be disti zuished: (i) a region of mixed $\mathrm{PC} / \mathrm{OG}$ micelles at low PC conce itration (white area. one macroscopic phase); (ii) a two-phase re -ion (black area), the upper (lighter) phase contains mixed micelles ([ $\mathrm{C}] \approx 1.3 \mathrm{~g} / \mathrm{l})$, the lower viscous coacervate phase containing most of the surfactant is called surfactant-rich phase (SRP); (iii) The third ic wn (area with stripes and dots) is a two-phase region similar as be:ore. but the upper phase additionaily contains vesicles, which can precipitate to form a white interphase band. The narrowing of the black stripes indicates that the amount of vesicles is increased at cost of the SRP. (iv) At $[O G]_{T} /[P C]_{T}<2$, the SRP vanished leaving one phase containing vesicles and - near to the two-phase region residual mixed micelles. In all samples $[O G]_{T}=27 \mathrm{~g} / \mathrm{l}$. The surfactant ratio $\mathrm{OG} / \mathrm{PC}\left(R_{\mathrm{T}}\right)$ refers to total $\mathrm{PC}$ and $\mathrm{OG}$; allowing for S-6 $g / 1$ monomeric OG (see Fig. 2) in the upper phase the ratio $R$ (SRP) in the SRP yields $90 \%$ of the value for total surfactants $R_{\mathrm{T}}$. The solution contained $10 \mathrm{mM}$ Pipes- $\mathrm{Na}(\mathrm{pH} 7.1$ ) and $150 \mathrm{mM} \mathrm{NaCl}$.

\section{Composition of the two phases}

Thin-layer chromatography (TLC) showed that OG and $\mathrm{PC}$ are present both in the upper and in the lower phase. The concentrations of $P C$ and $O G$ in the upper phase at $20^{\circ} \mathrm{C}\left(40^{\circ} \mathrm{C}\right)$ were measured to be $[\mathrm{PC}]=1.3$ $\pm 0.1 \mathrm{~g} / \mathrm{l}(1.3 \pm 6.1 \mathrm{~g} / \mathrm{l})$ and $[0 \mathrm{G}]=7.6 \pm 0.5 \mathrm{~g} / 1(6.1$ $\pm 0.5 \mathrm{~g} / \mathrm{l}$ ), respectiv sly. The [OG] value is slightly higher than the concentration of free OG measured by equilibrium dialysis (Fig. 2); the difference $(2 \pm 1 \mathrm{~g} / \mathrm{l})$ accounts for the $O G$ bound in micellar aggregates. In the lower phase (SRP), [OG] and [PC] are about the same: $42 \pm 1 \mathrm{~g} / \mathrm{l}$. Dilution of a sample taken from the upper phase with buffer solution is followed by a significant increase in turbidity. The turbidity increase correlates with the formation of vesicles as observed in the darkfield microscope **. Therefore, and with regard to the PC determination it can b: concluded that

\footnotetext{
- In contrast to micelles, vesicles can clearly be detected in the darkfield microscope. The appearance of vesicles aftez dilution ( $R$ becomes smaller) therefore indicates the presence of the phospholipid. The vesicle area in Fig. 3 is overexposed for better contrast of the beads in the middle.
} 
the clear upper phase (before dilution) contains detergent monomers and mixed micelles.

\section{Dependence of the miscibility gap on total surfactant} concentration

The extension of miscibility gap of the system OG-PC depends on the total surfactant concentration, respectively, on the mole fraction of water. The minimum value for the $\mathrm{PC}$ concentration at which the miscibility gap is macroscopically visible is in the range of $5 \mathrm{~g} / \mathrm{l}$. (A value of $2 \pm 0.5 \mathrm{~g} / 1$ was determined in the turbidity measurements, see also Ref. 10). At $[\mathrm{PC}]_{\mathrm{T}}=5.6 \mathrm{~g} /$ the corresponding value for $O G$ was $[O G]_{T}=9.1 \mathrm{~g} / 1$. With increasing concentrations of $O G$ and $P C$ the volume fraction of the SRP increased, until, at $[O G]_{T} \approx[P C]_{T}$ $=43( \pm 2) \mathrm{g} / 1$, the upper phase disappeared. Ai this point the ratio $R_{\mathrm{T}}^{\mathrm{c}}=[\mathrm{OG}]_{\mathrm{T}} /[\mathrm{PC}]_{\mathrm{T}}$ must be identical with this ratio in the SRP. When $[P C]_{T}=25 \mathrm{~g} / 1$ the values for $O G$ and $P C$ measured by dye reagents in the SRP are $[O G]=42 \mathrm{~g} / 1$. The higher value of $R_{\mathrm{T}}^{\mathrm{c}}$ in more diiuted samples is due to the $O G$ monomers in the upper aqueous phase (Table I).

\section{Temperature effects}

Temperature has only a small effect on the $R_{T}^{\mathcal{c}}$

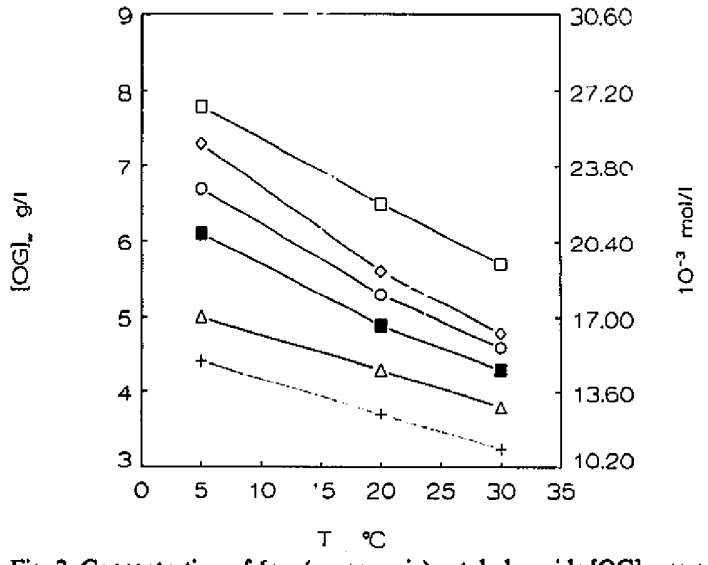

Fig. 2. Concentration of free (monomeric) octyl glucoside $[O G]_{w}$ as a function of temperature $T$. [OG $]_{\mathrm{w}}$ was determined by equilibrium dialysis at different values of $\left.R_{\mathrm{T}}=[\mathrm{OG}]_{\mathrm{T}} / \mathrm{PC}\right]_{\mathrm{T}} ;[\mathrm{PC}]_{T}$ was $22 \mathrm{~g} / \mathrm{l}$. The symbols correspond to different $[O G]_{T} ; 40 \mathrm{~g} / \mathrm{l}(\square), 30 \mathrm{~g} / \mathrm{l}(\diamond)$, $25 \mathrm{~g} / 1(0), 20 \mathrm{~g} / 1(\nabla), 10 \mathrm{~g} / 1(\Delta) \cdot[0]_{\mathrm{T}}=20 \mathrm{~g} / 1\left(R_{\mathrm{T}}=0.91\right)$ refers to the transition region (streaky area in Fig. 1). When PC95 was used instead of POPC, $[O G]_{w}$, was shifted to lower values $(+),[O G]_{T}=10$ $\mathrm{g} / \mathrm{l}$; only one curve is shown for clarity.

values (Fig. 1). However, separation of the phases was accelerated at higher temperatures. When the temperature was changed in the two-phase system, one phase

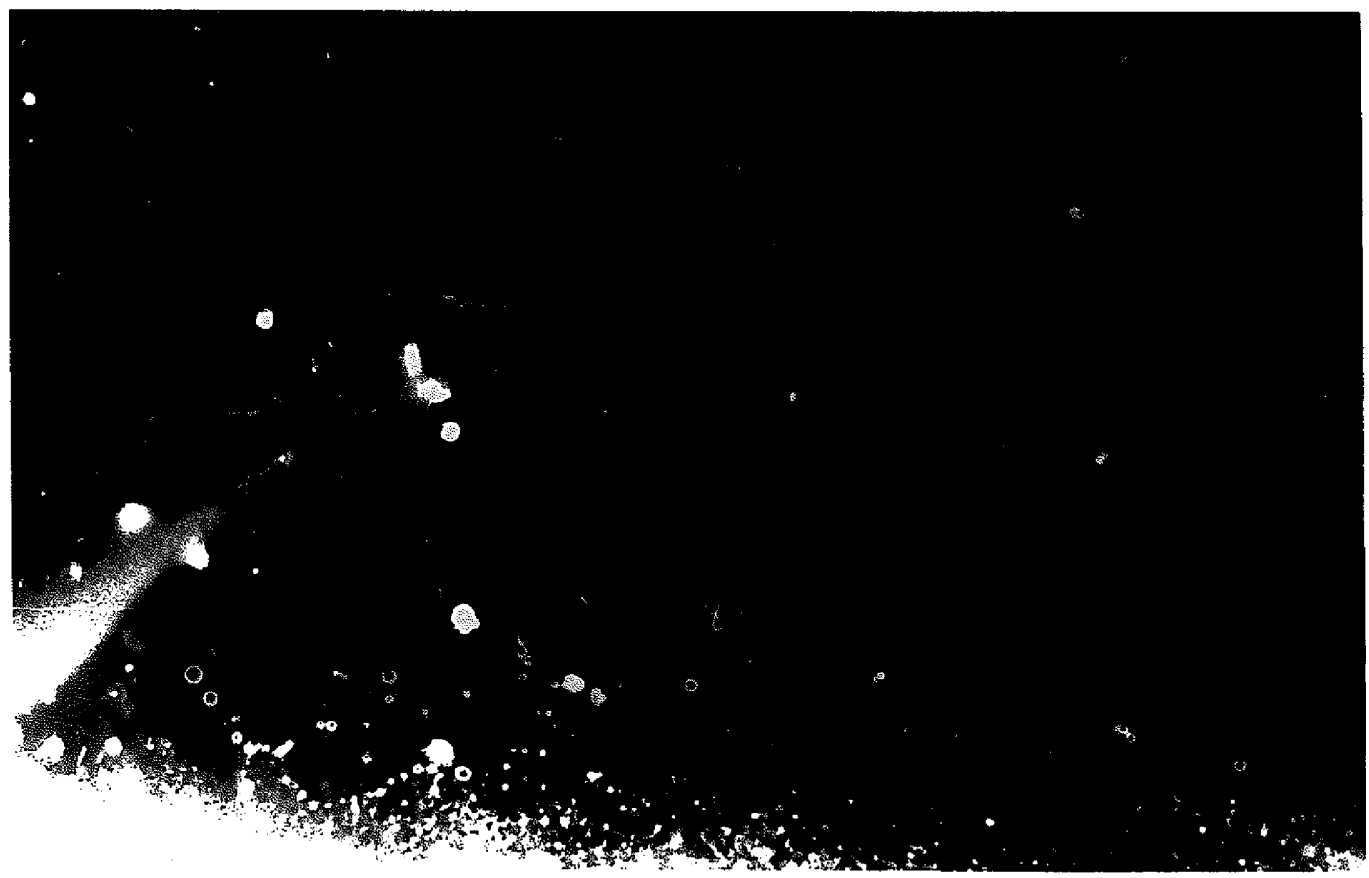

Fig. 3. Formation of the miscibility gap. The upper part is the surfactant-rich phase (SRP). The light band is the boundary of the phases. The lower light part contains vesicles, becoming less numerous towards the phase boundary (darker area in the middle). There, larger beads (spheres) arise by aggregation of smaller micellar lipid/detergent aggregates. Under the darkfield microscope it can be observed that the smaller spheres fuse to form the larger ones (diameter $\approx 5 \mu \mathrm{m}$ ), until they are merged in the SRP. The gradient is produced by evaporation of water from a vesicle solution

'.jandwiched between two slides) containing a subsolubilizing detergent concentration. The initial magnification was $100 \times,(24 \times 36 \mathrm{~mm})$. 
became turbid. When the system was cooled down the upper phase became turbid; conversely, upon heating the lower phase became opalescent. This increase in light scattering can be explained by the formation of either vesicles or droplets of a macroscopic phase depending on the direction of the temperature shift and the previous state of the system. The turbidity vanishes with time; the mixed phases separate again into two optically clear phases.

In order to get more information about the molecular changes following a temperature shift, we have measured the monomer concentration of octyl glucoside as a function of temperature. To measure the OG monomer concentration in equilibrium with the mixed OG/PC aggregates the samples were transferred into dialysis bags and dialysed against an equal volume of buffer containing $20 \mathrm{mM} \mathrm{OG}$ at the beginning $(20 \mathrm{mM}$ was chosen not to start too far from equilibrium; this and the small outer volume prevent larger changes of the $\mathrm{OG} / \mathrm{PC}$ ratio inside the dialysis bag). The results for five different concentrations of PC are shown in Fig. 2. Independent of the lipid concentration the concentration of OG monomers gets considerably lower towards higher temperatures. As the total OG concentration is constant, this leads to a slight increase of the $\mathrm{OG}$ bound in the aggregates.

\section{Microscopic phase formation}

The formation of the miscibility gap is illustrated by a phase boundary observed under a darkfield light microscope (Fig. 3). When a vesicle suspension, nearly saturated with OG, is kept below a cover glass the $O G$ concentration will rise with time due to the evaporation of water at the sides. Thus, a phase separation is induced. Between the vesicles and the phase boundary of the SRP there is a darker area (Fig. 3). Observing the development in this area one can seen small beads emerging at the boundary of the vesicle region. The beads grow by fusion until they merge into the SRP. The increase of the bead size corresponds to the decrease of turbidity in the dilution experiment. This (non-equilibrium) process is driven by the concentration gradient caused by the water evaporation on the SRP side.

When buffer (without any surfactant) was layered on top of a SRP phase, the reverse development was observed: large beads were budding off the SRP, becoming smaller with increasing distance from the phase boundary and finally resulting in a cloud of vesicies.

\section{Effect of different lipids on the phase separation}

Beside POPC different PCs, DOPC, DMPC and a PC from natural source, PC (95\%), all showed the phase separations. With DMPC the miscibility gap was shifted to smaller values of $\left.R_{\mathrm{T}}=[\mathrm{OG}]_{\mathrm{T}} / \mathrm{PC}\right]_{\mathrm{T}}$ below $20^{\circ} \mathrm{C}$. The critical ratio for DMPC is $R_{\mathrm{T}}^{\mathrm{c}}=0.95$ at $5^{\circ} \mathrm{C}$ in- stead of 1.05 for POPC. This shift in $R_{T}^{\mathrm{c}}$ coincides with the liquid-crystalline gel transition which has been observed for DMPC vesicles [20]. For PC (95\%) $R_{\mathrm{T}}^{\mathrm{c}}=1.2$ (Table I). In general the average chain length of the natural lipids is higher than that of POPC. PC (95\%) also shows a higher affinity to $O G$, according to the equilibrium measurements presented in Fig. 2.

Addition of charged lipids: whereas the addition of larger amounts of DPPS $(>10 \%)$ prevents the formation of a miscibility gap, lower concentrations result in streaks in the SRP, indicating a partitioning.

\section{NMR measurements}

${ }^{31}$ P-NMR measurements carried out to check for a possible lamellar or hexagonal structure in the SRP. yielded a narrow symmetrical peak. This result indicates that the emulsion is either isotropic or has a cubic structure [21!

\section{Partitioning of acetylcholine receptor}

Purified Torpedo californica acetylcholine receptor protein (n-AcChR) was added to the OG-PC suspensions at a protein concentration of $35 \mu \mathrm{g} / \mathrm{ml}$. Samples (of $1 \mathrm{ml}$ ) were prepared at two different lipid/detergent

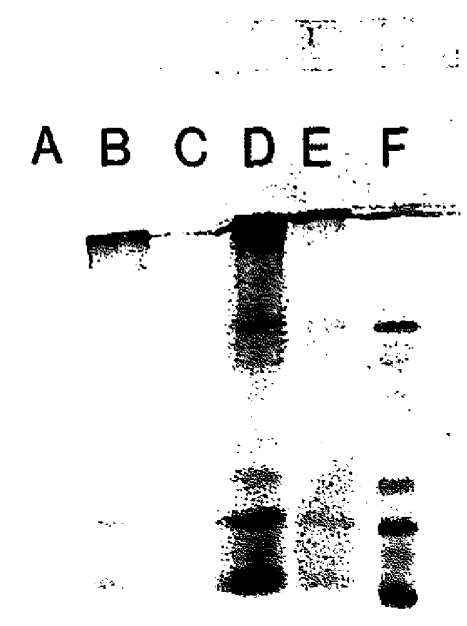

Fig. 4. SDS-PAGE of the $T$. cal $n-A c C h R$ in the different phases. Two different detergent/lipid ratios $R$ were used. In the first case $R$ is in the range of the miscibility gap. Slot B contains the protein from the lower phase (SRP), whereas no protein can be detected in the upper phase (slot $C$, water and $O G$ monomers). In the second experiment a lower $R$ value was chosen: vesicles formed on top of the SRP (middle phase, slot A-no protein). Slot D and E correspond to the lower and upper phase, respectively. Here little protein is recovered in the upper phase. Slot $F$ shows purified $n$-AcChR directly applied to the gel without further manipulation. 


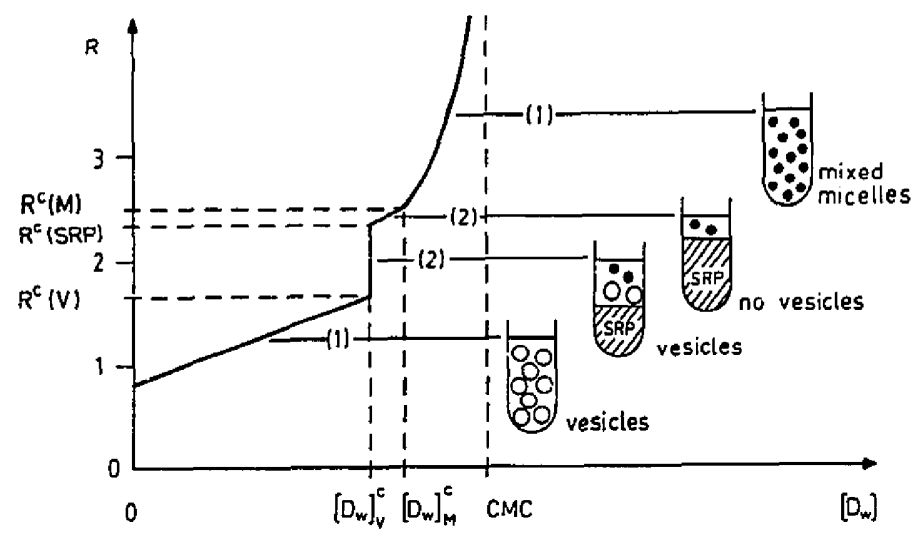

Fig. 5. Phase separations and structural transitions between lipid-detergent complexes. Extent of detergent incorporated in lipid structure $R$ as a function of free detergent concentration $\left[D_{w}\right]$. (1) and (2) represent one-phase and two-phase systems, respectively, as illustrated on the right hand side. The critical terms $R^{c}(V)$ of the vesicle phase and $R^{c}(S R P)$ of the surfactant-rich phase (SRP) describe the system at the critical value $\left[D_{w}\right]_{Y}^{c}$ in the miscibility gap. At $R=R^{c}(M)$ and $\left[D_{w}\right]=\left[D_{w}\right]_{M}^{c}$ the two-phase system changes to the one-phase mixed micelle phase. Obviously $\left[D_{w}\right] \leq C M C$, the critical micelle concentration of the pure detergent.

ratios; (i) in the miscibility gap (two phases) and (ii) at a higher lipid content, $R_{\mathrm{T}}=0.9$, where vesicles are on top of the SRP. After thoroughly mixing, the suspensions were left at $35^{\circ} \mathrm{C}$ to let the phase separation proceed $(\approx 1 \mathrm{~h})$. After phase separation, aliquots were taken from the different phases of each mixture and protein was precipitated by addition of $10 \mathrm{ml}$ of $90 \%$ cold ethanol (ice temperature). The precipitate was dissolved in SDS and analysed by SDS-PAGE. Fig. 4 shows a gel pattern of the different phases. Almost all protein is recovered in the lower, surfactant-rich phase. In the sample with vesicles little protein is recovered in the upper phase. There is no protein in the vesicles, indicating that there is no protein incorporation at this stage of vesicle formation. The band on top of the gel is aggregated protein, probably resulting from the ethanol precipitation.

\section{Theoretical approach}

Lichtenberg has developed a theoretical approach for the solubilization of bilayers [7]. It is generally assuined that the detergent molecules incorporated in the lipid aggregates $\left(D_{b}\right)$ are in equilibrium with the free, monomeric detergent $\left(D_{w}\right)$ in solution. The extent of detergent incorporation in lipid detergent complexes is defined by the ratio

$$
R=\left[\mathrm{D}_{\mathrm{b}} \mathrm{l} / \mathrm{L}\right]_{\mathrm{T}}
$$

where $[L]_{T}$ is the total concentration of the lipid. Denoting by $[D]_{T}$ the total detergent concentration, mass conservation yields

$$
\left[D_{w}\right]=[D]_{T}-R \cdot[L]_{T}
$$

When the partition coefficient of the detergent between the aqueous phase $D_{w}$ and the lipid structure $D_{b}$

$$
\Gamma=\left[\mathrm{D}_{\mathrm{b}}\right] /\left[\mathrm{D}_{\mathrm{w}}\right]
$$

is constant, the incorporation ratio

$R=(\Gamma /[\mathrm{L}] \tau)\left[D_{\mathrm{w}}\right]$

is linearly dependent on $\left[D_{w}\right],[6]$.

It was found that bilayer vesicle solubilization starts at a critical value $R^{c}(\mathrm{~V})=1.3-1.8(\mathrm{~mol} / \mathrm{mol})$ of the $\mathrm{OP} / \mathrm{PC}$ system [6]. The vesicles are transformed to mixed micelles associated with the ratio $R^{c}(M) \approx 2.6$, indicating a higher affinity of the detergent for the mixed micelles compared with vesicles. In the $[D]_{T}$ range of vesicle solubilization both the compositions of the vesicles, $R^{\mathrm{c}}(\mathrm{V})$, and of the micelles, $R^{\mathrm{c}}(\mathrm{M})$, remained constant [6].

The formation of vesicles from mixed micelles and the solubilization of vesicles which are summarized in our phase diagram (Fig. 5) ${ }^{*}$, may be also described in terms of critical ratios $R^{c}$. We have found that both $R^{\mathrm{c}}(\mathrm{V})$ and $R^{\mathrm{C}}(\mathrm{M})$ characterize phase separations. A third critical ratio $R^{\mathrm{C}}(\mathrm{SRP})$, within the miscibility gap, defines the upper limit $R$ were vesicles can occur.

When increasing amounts of detergent $D$ are added to lipid vesicles, $R(V)$ increases up to a critical point

\footnotetext{
* The schematic phase diagram in Fig. 5 was constructed to visualize the proposed changes of $R$ and $\Gamma$ around the transition region. Therefore, as a first approximation $\Gamma$ was taken as constant below the phase separation, respectively, the vesicle-micelle transition. However, it should be noted that there is also a change in $\Gamma$ at 4 $\mathrm{mM}[\mathrm{OG}]_{w}$ as recently published by $\mathrm{M}$. Ueno [27]. Of course, the transition in the 'real' curve is more smooth than depicted here.
} 
$R^{\mathrm{c}}(V)$ where the concentration of free detergent is $\left[\mathrm{D}_{\mathrm{w}}\right]_{\mathrm{v}}^{\mathrm{c}}$. Further addition of $D$ gives rise to the formation of micelles. When the micellar concentration reaches a critical value $[\mathrm{M}]^{\mathrm{c}}(\approx 1.3 \mathrm{~g} / 1 \mathrm{PC}$, value measured in the upper phase) a macroscopically separated phase appears at the bottom of the solution (see also Results: Dependence of the miscibility gap on total surfactant concentration, Fig. 1 and Ref. 10). This surfactant-rich phase (SRP) is formed by aggregation, respectively, fusion of micelles exceeding a critical value $[\mathrm{M}]^{\mathrm{c}}$; see also Discussion and Fig. 3. Upon further addition of D. the SRP increases in size at the expense of vesicles in the upper phase. The added detergent is used to dissolve the vesicles along the $R^{\mathrm{c}}(\mathrm{V}) \rightarrow R^{\mathrm{c}}(\mathrm{SRP})$ transition, such that the concentration of 'free' detergent remains sonstant at $\left[D_{w}\right]_{\mathrm{v}}^{\mathrm{c}}$. At $R=R^{\mathrm{c}}(\mathrm{SRP})$ there are no more vesicles in the upper phase of the two-phase system. Further addition of detergent will increase $\left[D_{w}\right]$ and $R(S R P)$ igain. Therefore, size, form and surface properties of the remaining micellar aggregates alter, to , until at $\left[D_{w}\right]=\left[D_{w}\right]_{M}^{C}$ the two phases change into one phase of mixed micelles. Thus between the two critical values $R^{\mathrm{c}}(\mathrm{V}) \approx 1.5$ at $\left[\mathrm{D}_{\mathrm{W}}\right]_{\mathrm{V}}^{\mathrm{c}}$ and $R^{\mathrm{c}}(\mathrm{M}) \approx 2.6$ at $\left[\mathrm{D}_{\mathrm{w}}\right]_{\mathrm{M}}^{\mathrm{c}}$ the miscibility gap exists, in which, at $R=R^{\mathrm{c}}(\mathrm{SRP})$, the (microscopic) vesicle phase disappears. (The $R^{\mathrm{c}}$ values were taken from Fig. 1 and corrected for the monomeric OG).

When vesicles are formed from mixed micelles the sequence of steps is reversed. At $R \leq R^{\mathrm{c}}(\mathrm{M})$ two phases appear, at $R \leq R^{\mathrm{c}}(\mathrm{SRP})$ vesicles are formed in the upper phase and, finally, at $\left[D_{w}\right] \leq\left[D_{w}\right]_{v}^{c}$, the SRP and micelles in the upper phase have disappeared and a homogeneous vesicle phase is formed.

\section{Discussion}

The solubilization of lecithin by $O G$, reported by Jackson et al., is accompanied by a large increase in the optical turbidity [6]. The authors presumed that this phenomenion is due to vesicle aggregation. Obviously, in the light of our data, they have measured the beginning of a phase separation. The separation is slow below $25^{\circ} \mathrm{C}$ and very slow (up to several hours) when the SRP occurs together with vesicles (streaky area in Fig. 1). Therefore the phase separation may easily be overlooked.

\section{$O G / P C$ ratio and phase separation}

In order to analyse the composition of the micelles and of the vesicles these two forms of aggregates were separated by differential centrifugation [6]. Our data, and also Ref. 10 show that actually there is not a direct transformation from vesicles to micelles at $[P C] \geq 2$ mM. Rather, the transition occurs via a SRP. It is easy to optically monitor the transition due to the phase separation; centrifugation is not necessary. Interest- ingly, the OG/PC ratio which was measured for the micelles at th: transition. $P=2.6$ [6], compares with that we have "ound for the xistence of the two phase region (SRP- to vesicles). $i l=2.4-2.6$. This range is somewhat lover than the dita reported by Ollivon et al., $R=3.0[1)]$. Likewise, the break point $\mathrm{B}(R=2.1)$ they determined by resonance energy transfer (RET) and optical density is even higher than the OG/PC ratio that marks the boundary between vesicles and vesicles + SRP $\left(R^{\mathrm{c}}(\mathrm{SRP})=1.7 \pm 0.1\right.$, left side in Fig. 1). Considering that the SRP forms by aggregation of micelles, the latter must occur at $R^{\mathbf{c}} \leq 1.7$. Separation of vesicles and micelles by centrifugation yielded $R^{\mathrm{c}}$ values in the range of $1.3 \leq R^{\mathrm{c}} \leq 1.8$, depending on the size of the vesicle [6]. The white vesicular band which occurs ibove $R=1.7$ (streaky area in Fig. $1, R_{\mathrm{T}}^{\mathrm{c}} \approx 2.0$ ) is likely to consist of open bilayers sheets as suggested [10]. The different value for the onset of micelle formation $[6,11\}$ and our data -ompared to [10] could be due to different lipid preparations. We have found, hat already small amounts of negatively charged lipid abolished the phase separation. An important difference in the realization of the measurements was that Ollivon et al. [10] recorded their data continuously,i.e., not in equilibrium. Interestingly, the maximum which marks break point $B$ [10] does not occur in the turbidity measurements by Paternostre et al. [11]. A maximum did not occur in our turbidity measurements either (data not shown). Anyhow, a change in a spectroscopic quantity like a turbidity maximum does not necessarily mean that there is a phase transition or phase separation.

Negative lipids will increase the electrostatic repulsion as well as influence the curvature of detergentlipid aggregates. PC (95\%) showed a significantly higher $\Gamma(O G)$ value compared with that of POPC (see Fig. 2).

Ollivon et al. [10] have measured a $[\mathrm{OG}] /[\mathrm{PC}]$ value in the upper phase which is characteristic for a lamellar structure. They also report the upper phase to be turbid. This would correspond to the streaky area in our phase diagram (Fig. 1). There the SRP is in equilibrium with vesicles or lamellar sheets in the upper phase. However, we could not detect vesicular structures in the upper phase, when the samples separated into two clear phases (see 2nd footnote on n. 227).

\section{Dependence of phase separation on total surfactant con- centration}

No phase separation was found when $[\mathrm{PC}] \leq 2 \mathrm{~g} / \mathrm{A}$ PC [10]. This compares rather well with the PC concentration $(1.3 \mathrm{~g} / \mathrm{l})$ we have measured in the upper phase (without vesicles). Obviously there is a minimum concentration of micelles, $[\mathrm{M}]^{\mathrm{c}}$, necessary to form the SRP. The NMR measurements, the relatively high water content and the X-ray data [10] suggest that the SRP consists of loosely aggregated micellar structures. The 
aggregation of micelles to the SRP is comparable to the formation of micelles from detergents at the CMC; it was named superaggregation in a different system of two surfactants [22]. The upper limit of the phase separation is reached when the total composition matches with that in the SRP. The composition at that point, $[O G]_{\mathrm{T}} \approx[\mathrm{PC}]_{\mathrm{T}}=43 \pm 2 \mathrm{~g} / 1$ compares well with $[O G]_{T}=42 \mathrm{~g} / 1$ and $[\mathrm{PC}]_{\mathrm{T}}=42 \mathrm{~g} / 1$ measured in the SRP at $[P C]_{T}=25 \mathrm{~g} / 1$. The difference of $\pm 2 \mathrm{~g} / 1$ mainly accounts for the difference between the $R^{\mathrm{C}}(\mathrm{M})$ and $R^{\mathrm{c}}(\mathrm{SRP})$ values. The increasing value of $R_{\mathrm{T}}^{\mathrm{c}}$ towards lower total surfactant concentrations (Table 1) corresponds to the additionai amount of free (monomer) $O G$ in the larger upper phase $(\approx 14 \mathrm{mM}=4.1 \mathrm{~g} / \mathrm{l})$.

\section{Theoretical approach and temperature dependence of $S R P$ formation}

The formation of the coacervate phase by Triton $\mathrm{X}-114$ is accompanied by the separation of one molecule $\mathrm{H}_{2} \mathrm{O}$ per detergent molecule [23]. This will change the surface free energy [22] and therefore allow the micellar-lamellar-transition leading to the dense lower phase. Generally, the structures surfactant molecules can assume are determined by a surfactant parameter $v /(a \cdot l)$, the volume of the molecule $(v)$ divided by the product of headgroup area $(a)$ and length $(l)$ [24]. In the case of $O G$ and $P C$ it is clear that addition of $P C$ increases the mean value of $v /(a \cdot l)$ and accordingly the probability of lamellar structures, or intermediate structures.

On the same line, from the phase diagram in Fig. 1 it is expected that $R(\mathrm{M})$, the $\mathrm{OG} / \mathrm{PC}$ ratio in the micellar structures near to the transition, decreases at higher temperature. On the contrary, the determination of free $O G$ in equilibrium with the lipid bound form shows an increase in $\Gamma$ and therefore in $R(\mathrm{M})$, too. However, an increase in $v /(a \cdot l)$ due to increased motion of the hydrocarbon chains can account for the observed shift of $R_{\mathrm{T}}^{\mathrm{c}}$. This interpretation is supported by the shift of $R_{\mathrm{T}}^{\mathrm{c}}$ in the opposite direction when DMFC was used below $T_{\mathrm{c}}$ (gel state) instead of POPC.

According to previous results $[6,10]$ and in line with out data there is a 'jump' in $R\left(R^{\mathfrak{c}}(\mathrm{V}) \rightarrow R^{\mathrm{c}}\right.$ (SRP), see Fig. 5) without measurable change in $\left[D_{w}\right]$. Raoult's law, requiring ideal mixing, is not appropriate to calculate the concentration of the free detergent $\left[D_{w}\right]$ within the transition range as was proposed by Ref. 10. Rather, the lamellar and the micellar structures can be treated as different phases with corresponding different partition coefficients $\Gamma$ for D. Thus, Eqn. 4 has to be applied separately to the different phases, at least.

\section{Applications of the phase separation}

Phase separation conditions may be applied to separate different proteins, as achieved with Triton X-114 [14]. The detergent Triton X-114 shows a miscibility gap above $28^{\circ} \mathrm{C}$, the critical point. In the two-phase system of Triton X-114, membrane proteins are concentrated in the lamellar, detergent-rich phase. Here we have shown that the T. cal. n-AcChR partitions into the SRP of appropriate PC/OG mixtures. An important difference between Triton and OG is the structure of the SRP. Whereas the SRP of Triton X-114 shows a lameilar structure in NMR measurements and freeze-fracture electronmicroscopy, ne such structure was seen in the SRP of PC/OG mixtures. On the basis of X-ray scattering data, an 'unoriented ordered' structure was suggested [10].

It is well known that the addition of lipid to detergent-solubilized membrane proteins can maintain the native structure of the proteins during purification and reconstitution. Therefore, the $\mathrm{PC} / \mathrm{OG}$ miscibility gap can serve as a tool for protein purification under relatively mild conditions.

\section{Reconstitution of membrane proteins}

There is no question that the phase separation has its own implications on the reconstitution of membrane proteins. Especially, the kinetics of detergent removal from the SRP is very slow. Nevertheless the OG/FC system can be used as a model system for reconstitution, where the microscopic micellar 'phase' is changed into a macroscopic phase and therefore facilitates the analysis of vesicle formation and protein incorporation. It was found [25] that the n-AcChR reconstituted by detergent dialysis was concentrated in some vesicles, whereas the other vesicles remained protein free. This observation can be rationalized in terms of our phase diagram. There is no protein incorporation at the beginning of vesicle formation, where the protein remains in the SRP. Vesicles are favorably formed from the micelles in the upper phase. In a later stage of dialytic dilution the detergent content is so low that protein-lipid interactions are dominant and protein-rich vesicles are formed. For the analysis of ion channels in a planar bilayer, however, favorably one or a few channel proteins should be present in a lipid vesicle. At present this is still difficult to achieve. Preliminary data (not shown) suggest that only at $[\mathrm{PC}]<1 \mathrm{~g} / 1$ during dialytic detergent removal, vesicles of low protein content are formed. On the other hand, fast dilution of a protein/lipid/ detergent solution is unlikely to incorporate the protein into vesicles. It was found that receptor monomers and dimers can be separated in the presence of lipids on a sucrose gradient containing no detergent [26]. Receptor monomers and dimers incorporated into vesicles cannot be separated on a sucrose gradient. Obviously, the fast removal of the detergent prevents protein/lipid vesicle formation.

In any event, the data of the macroscopic phase separation of the OG/PC system are instrumental to 
select favorite conditions fo: vesicle formation and for low copy number protcin incorporation into vesicles.

\section{Acknowledgements}

We thank Dr. Peter M. MacDonald and Prof. Dr. Joachim Seelig for trie ${ }^{31} \mathrm{P}-\mathrm{NMR}$ measurements. We thank also Birgit Büddemann for excellent technical assistance and $\mathrm{Dr}$. R. Schomäcker for discussion of the manuscript. We gratefully acknowledge financial support by the Deutsche Forschungsgemeinschaft (SFB 223/C1, C2 to E.N. and T.S.)

\section{References}

1 Helenius, A. and Simons, K. (1975) Biochim. Biophys. Acta 415. 29-79.

2 Tanford, C. and Reynolds, J.A. (1976) Biochim. Biophys. Acta 457, 133-170.

3 Lindsirom, J., Anholt, R., Einarson, B., Engel, A., Osame, M. and Montal, M. (1980) J. Biol. Chem. 255, 8340-8450.

4 Montal, M., Darzon, A. and Schindler, H. (1981) Q. Rev. Biophys. 14, 1-79.

5 Schürholz, T. and Schindler, H. (1983) FEBS Lett. 152, 187-190.

6 Jackson, M.L., Schmidt, C.F., Lichtenberg. D., Litman, B.J. and Albert, A.D. (1982) Biochemistry 21, 4576-4582.

7 Lichtenberg. D. (1985) Biochim. Biophys. Acta 821, 470-478.

8 Fromherz, P. and Ruppel, D. (1985) FEBS Lett. 179, 155-159.

9 Schürholz, T. (1987) Biol. Chem. Hoppe-Seiler 368, 1270 (Abstr.)

10 Ollivon, M., Ejdelman, O., Blumenthal, R. and Walter, A. (1988) Biochemistry 27, 1695-1703.
11 Paternostre, M.-T., Roux, M. and Rigaud, J.-L. (1988) Biochemistry 27, 2668-2677.

12 Robinson. B.H. (1986) Nature 320, 309; Fletcher, P.D.1. Freedman, R.B., Robinson, B.H. Rees, G.D. and Schomicker, R. (1987) Biochim. Biophys. Acta 912, 278-282.

13 Kopp. F.. Meyer, H.E. and Reinauer, H. (1985) Biol. Chem. Hoppe-Seiler 366, 695-698.

14 Bordier, C. (1981) J. Biol. Chem. 256. 1604-1607.

15 Schurholz, T., Weber. J. and Neumann, E. (1989) Bioelecirochem. Bioenerg. 21, 71-81.

16 Chang. H.W. and Bock. E. \{1977) Biochemistry 16, 4513-4520.

17 Schindler, H., Spillecke. F. and Neumann, E. (1984) Proc. Natl. Acad. Sci. USA 81, 6222-6226.

18 Itaya, K. and Ui. M. (1966) Clin. Chim. Acta 14, 361-366.

19 Scott, T.A. and Melvin, E.H. (1953) Anal. Chem, 25, 1956; Dische, Z. (1962) Methods in Carbohydrate Chemistry, Academic Press, 1. 488.

20 Metcalfe, J.C. (1975) in Functional Linkage in Biomolecular Systems (Schmitt. F.O., Schneider. D.M. and Crothers, D.M., eds.), Raven Press, New York.

21 Shyamsunder. E., Gruner. S.M. Tate. M.W., Turner, D.C., So, P.T.C. and Tilcock, C.P.S. (1988) Biochemistry 27, 2332-2336.

22 Evans, D.F. and Ninham, B.W. (1986) J. Phys. Chem. 90. 226-234

23 Heusch. R. (1984) Ber. Bunsenges. Phys. Chem. 88, 1093-1098.

24 Israelachvili, J.N., Mitchell. D.J. and Ninham. D.W. (1976) J. Chem. Soc. Faraday Trans. 2 72, 1525-1568; Wennerström. H. and Lindman, B. (1979) Phys. Rep. 52, 1; Mitchell, D.J. and Ninham, B.W. (1981) J. Chem. Soc. Faraday Trans. 2 77. 601-629.

25 Anholt, R., Lindstrom. J. and Montal, M. (1981) J. M. Biol. Chem. 256, 4377-4381.

26 Volz. V. (1987) Diploma Thesis. University of Bielefeld.

27 Ueno. M. (1 $\$ 89)$ Biochemistry 28, 5631-5634. 